\title{
LO QUE EL OJO NO VE: UN ESTUDIO DE CASO BASADO EN PROCESOS DE INDAGACIÓN CO(WIKI)LABORATIVOS
}

\author{
(WHAT THE EYE DOES NOT SEE: AN INQUIRY (COWIKI-) BASED LEARNING CASE STUDY)
}

Iván M. Jorrín Abellán

Rocío Anguita Martínez

Bartolomé Rubia Avi

Inés Ruíz Requies

Sara Villagrá

Facultad de Educación y Trabajo Social. Universidad de Valladolid (España)

Yannis Dimitriadis

Jose Antonio Marcos

Escuela técnica Superior de Ingenieros de Telecomunicación de Valladolid

(España)

\section{RESUMEN}

El proceso de convergencia al que se están enfrentando las universidades europeas en estos momentos, requerirá de cambios metodológicos sustanciales que provean nuevos entornos de enseñanzaaprendizaje a los estudiantes del siglo XXI. La tradicional enseñanza presencial se verá apoyada por el uso de tecnologías de la información y la comunicación (TIC). Para dar respuesta a tan acuciante situación, se ha diseñado, puesto en práctica y evaluado una experiencia innovadora en la asignatura (presencial) de Nuevas Tecnologías Aplicadas a la Educación en la Facultad de Educación y Trabajo Social de la Universidad de Valladolid (España). El artículo que proponemos presenta un estudio de caso realizado durante el presente curso académico 2006-2007. El diseño de la asignatura se ha nutrido de dos aproximaciones teórico-prácticas diferentes pero complementarias. En primer término, el proceso se asienta en los planteamientos establecidos dentro del campo del Computer Supported Collaborative Learning (CSCL) (Koschman, 1996), para mediar procesos de enseñanza-aprendizaje en un entorno de educación superior presencial. En segundo lugar, el proceso se apoya en una metodología participativa basada en el Inquiry based Learning (IBL, aprendizaje por indagación) (Bruce, 2000). Para unir ambas propuestas se ha empleado la tecnología wiki, generándose un entorno especial de colaboración que hemos denominado "Inquiry (cowiki-) based learning" (Aprendizaje basado en la co(wiki)laboración). La puesta en marcha y evolución de la innovación dentro de la asignatura ha sido evaluada por una comunidad de práctica transdisciplinar, formada por investigadores-docentes provenientes del campo 
de la tecnología y la educación. Este artículo enfatiza, a su vez, el valor de analizar escenarios CSCL mediante el empleo de metodologías de investigación cualitativas que muestren evidencias acerca de los modelos participativos de indagación propuestos en la asignatura de Nuevas Tecnologías.

Palabras clave: CSCL, estudio de casos, aprendizaje por indagación, TIC

\begin{abstract}
In this paper we present a case study of an undergraduate $I C T^{1}$ course at the College of Education in the University of Valladolid (Spain). Two different but complementary approaches have been put in practice in the design of the course. Firstly, it is based on the principles of the Computer Supported Collaborative Learning (CSCL) (Koschman, 1996), field, using technologies to support the collaborative learning process. On the other hand, it follows an Inquiry based Learning methodology. This special mixture offers a "tasty dish" we have called "Inquiry (cowiki-) based learning". The whole process was designed, implemented and evaluated by a transdisciplinary community of practice formed by many researchers coming from the ICT and education fields. This paper emphasizes the value of studying CSCL scenarios by using qualitative methods, showing evidence about participatory models of inquiry.
\end{abstract}

Key words: CSCL, case study, wiki, inquiry based learning, ICT

\title{
CONOCIÉNDONOS
}

Su acento delata su procedencia. Sus expresiones sagaces y su manera de hablar, pronto nos indican que Rocío proviene de las tierras del sur. Es profesora titular del departamento de Pedagogía y dispone de experiencia docente en el campo de las Nuevas tecnologías aplicadas a la educación, acumulada durante los más de 10 años que lleva impartiendo la citada asignatura en la Universidad de Valladolid. Durante el curso académico 2005-2006, después de poner en marcha y obtener resultados de evaluación de una experiencia de innovación previa dentro de su asignatura (RubiaAvi et al, 2006), comenzó a madurar nuevas propuestas que ayudasen a mejorar los puntos débiles mostrados por la evaluación.

La innovación mencionada, desencadenante de la que nos ocupa, se basaba en los principios del Computer Supported Collaborative Learning (CSCL). La asignatura de Nuevas Tecnologías Aplicadas a la Educación (NNTT) se desarrollaba como un proyecto completo y complejo al que el alumnado debía ir dando respuesta a lo largo del curso académico. La asignatura, siguiendo las líneas de la declaración de Bologna (Bologna, 1999), estaba plenamente centrada en el alumno, dando especial relevancia al uso de un Learning Managment System (LMS) de apoyo a la evolución de los aprendizajes y a los procesos de tutoría individualizada. El diseño educativo fue evaluado y se obtuvieron algunas conclusiones a partir de las cuales surgió la 
experiencia que describimos en este artículo. Algunas de estas cuestiones particulares se pueden resumir en:

- el diseño educativo generaba un exceso de carga de trabajo en el alumnado y en el profesorado.

- La distribución del alumnado en grupos de trabajo heterogéneos cambiantes dificultaba el devenir de la asignatura.

- el alumnado no disponía de un guión de aprendizaje, más allá del programa de la asignatura, que le guiase a lo largo del complejo diseño de aprendizaje colaborativo.

- El diseño de la asignatura era muy extenso en su parte teórica, minimizando el tiempo que el alumnado podía dedicar a la aplicación práctica de los contenidos teóricos trabajados.

- Los recursos tecnológicos de apoyo empleados no se entendían como una unidad; lo que dificultaba su uso por parte del alumnado.

B: iEstá claro que hay cosas que debemos cambiar! Lo bueno de evaluar es que ahora, por lo menos, tenemos una idea de lo que mejor y peor ha funcionado.

R: Sí, sí. Creo que hay que pensar en "darle una vuelta" al diseño de la asignatura. Hay que simplificar unas cosas, y vamos a tener que prescindir de otras. Os propongo que revisemos los datos que tenemos de las observaciones y los grupos de debate, y que después hagamos propuestas concretas.

B: Me parece bien. Nos damos una semana si te parece, y nos volvemos a juntar.

Fueron numerosas las discusiones que Rocío mantuvo, junto a los dos compañeros de departamento con los que comparte asignatura, para tratar de superar los problemas generados por el diseño de la asignatura. No obstante, éstas fueron muy fructíferas y promovieron el diseño que describimos en el apartado siguiente. Éste sigue la línea de trabajo marcada en los últimos años por el grupo de investigación transdisciplinar GSIC-EMIC ${ }^{2}$, al que pertenecen Rocío y sus compañeros. Las innovaciones metodológicas puestas en marcha en la asignatura de Nuevas Tecnologías siempre se han realizado en el seno de esta comunidad de práctica. Es ésta una cuestión de máxima relevancia, que además de proveer un entorno asentado de colaboración entre profesionales de distintas áreas, tiende puentes y facilita la puesta en marcha de nuevos recursos educativos de marcado carácter tecnológico tanto en los momentos educativos como en los evaluativos de la experiencia que nos ocupa. 


\section{APLICANDO LO APRENDIDO}

R: He estado revisando mis notas del encuentro que tuve con Chip, y creo que podríamos generar un diseño de asignatura a medio camino entre los planteamientos del CSCL y las ideas del pragmatismo y el aprendizaje por indagación.

B: La verdad es que la experiencia que lleva Chip es muy motivante. Yo también he estado consultando la web de su proyecto, y me parece que podemos hacer algo.

R: ¿Podríamos aplicar el ciclo de la indagación a nuestro curso?

B: Creo que sí, pero hay que ver de qué manera lo hacemos. Hay que pensar también en la tecnología de apoyo que vamos a usar. "ilabs"

$R:$ Sí, sí, está claro. Él, usa un software que han diseñado en su universidad, el

B: y... ¿Podríamos utilizarlo nosotros?

R: No, es propietario...

Los posicionamientos teórico-educativos tanto de Rocío como de sus compañeros en la asignatura y en el grupo de investigación al que pertenecen, se acercan al socio-constructivismo, por lo que los procesos de construcción colaborativa de conocimiento son de máxima relevancia en su asignatura. Teniendo esta cuestión presente, podemos entender que uno de los pilares teóricos fundamentales sobre los que se asienta la innovación propuesta, sea el CSCL. No obstante, tal y como muestra el diálogo al inicio de esta sección, el profesorado de la asignatura se vio en la necesidad de profundizar en este aspecto para encontrar nuevas fórmulas para fomentar la colaboración entre el alumnado, huyendo de diseños educativos complejos. Por ello, tras una reflexión extensa, y después de varios encuentros con el profesor Bertran C. Bruce ${ }^{3}$, se decidieron por unir en un mismo diseño educativo los planteamientos del CSCL y los del IBL (aprendizaje por indagación).

Esta unión se produjo porque, tanto Rocío como sus compañeros entendían que los principios del IBL como los del CSCL se basan en una manera común de entender el proceso educativo, aunque sus variantes respecto al tipo de entornos donde se desarrollan puedan ser diferentes. Entre otras cuestiones comparten:

- Ambos planteamientos pedagógicos entienden quela educación ha de plantearse de manera global respecto de las áreas que concurren a la hora de planificar y realizar las actividades educativas.

- También asumen que dichas actividades deben estar apoyadas en situaciones reales o cercanas, porque como entiende Rocío, apoyándose en las teorías 
mediacionales de la psicología (Pérez Gómez, 1992), una persona aprende mejor desde los contenidos que tienen significado concreto en su mundo o que de alguna manera conceptualiza, construyendo conceptos nuevos sobre los que ya posee.

- A su vez, comparten la forma de plantear los aprendizajes, formulando no tanto estructuras formales de contenidos sino preguntas sobre situaciones entendidas como problemas, es decir, acercándose al mundo desde una perspectiva problematizadora.

- Otro aspecto en el que concurren estas dos corrientes es la forma en que se plantean las tareas de aprendizaje, pensando que la mejor forma de realizar el proceso de aprendizaje es construyendo ideas personales que ayuden a reconstruir los conceptos aprendidos (Grundy, 1991) dentro de cada persona, en vez de repetir mecánicamente dichos conceptos objeto de aprendizaje; asumiendo, de esta forma, el proceso de integración de conceptos y esquemas mentales de la realidad por medio de la reflexión.

- Y por último, ambas corrientes conciben de una manera más o menos extendida que el proceso de trabajo para un buen desarrollo del aprendizaje, se ha de producir necesariamente por medio de la relación social de las personas que aprenden juntas, considerando el trabajo en grupo y la participación activa de los educandos, como algo fundamental para su educación.

Estas características comunes a ambos planteamientos presentan numerosas similitudes con la forma en que Rocío y sus compañeros entienden la asignatura que les ocupa. De esta manera, consideran que la formación en NNTT del alumnado de magisterio debería estar centrada en la formación práctica para realizar diseños curriculares ajustados, usando los recursos tecnológicos como un medio efectivo que facilite las actividades previstas en el diseño, no como suele ser habitual, paralizándolas, trasformándolas o limitándolas. Para que esta situación se produzca, un educador debe tener, además de un conocimiento efectivo de los recursos tecnológicos a nivel de usuario, la capacidad de diseñar o apoyar el diseño y desarrollo de los recursos tecnológicos necesarios para su práctica docente.

Por este motivo, Rocío y sus colaboradores unieron las potencialidades tecnológicas de los entornos CSCL con las posibilidades educativas del aprendizaje por indagación. 
Siguiendo estos planteamientos se diseñó la asignatura de NNTT para un grupo de 46 alumnos/as del primer curso de la titulación de Educación Social en el primer semestre del curso académico 2006-2007.

R: Tenemos que reconstruir el programa convirtiendo los puntos clave de cada tema relevante en preguntas a las que el alumnado vaya dando respuesta. ¿Cómo lo veis?

I: Sí, sí, creo que lo mejor será repartirnos dos temas cada uno e ir viendo las posibles preguntas que van saliendo. Nos damos un plazo de quince días y lo discutimos.

B: Me parece bien, pero a la vez deberíamos ir pensando tanto en lo que le vamos a pedir al alumnado, como en los materiales que les vamos a ir dando para desarrollar las preguntas que planteemos.

I: iDe acuerdo! También voy a ir mirando la tecnología que podemos utilizar para un diseño de estas características.

$R$ : Entonces, yo miro un tema más y así tú tienes tiempo de ir analizando la tecnología de soporte que puede servirnos.

I: iEstupendo!

Como puede extraerse del diálogo anterior mantenido por Rocío y sus compañeros, el proceso de rediseño de la asignatura fue laborioso y requirió de dos meses de trabajo en equipo. Se tomó como punto de partida el programa impartido hasta ese momento y desde ahí se fue construyendo un esquema de trabajo repartido en temas, siguiendo siempre el esquema tradicional de los procesos IBL (Figura I). De esta forma, se propusieron seis temas divididos en cinco fases comunes: Pregunta, investiga, crea, discute y reflexiona. La primera fase está formada por una o varias preguntas que guían el trabajo a través del tema. No tienen una respuesta única ni correcta, sino que son de carácter abierto, promoviendo así el debate y la reflexión sobre el contenido a tratar. La segunda fase, investiga, está compuesta por una serie de materiales (artículos, presentaciones, etc.) que ayudan al alumnado a dar respuesta a la pregunta de la etapa anterior. Posteriormente el alumnado debe crear un artefacto fruto de su investigación, para que en la cuarta fase sea criticado por sus iguales. El proceso finaliza con una reflexión personal alrededor de los aprendizajes más significativos alcanzados en cada tema. 


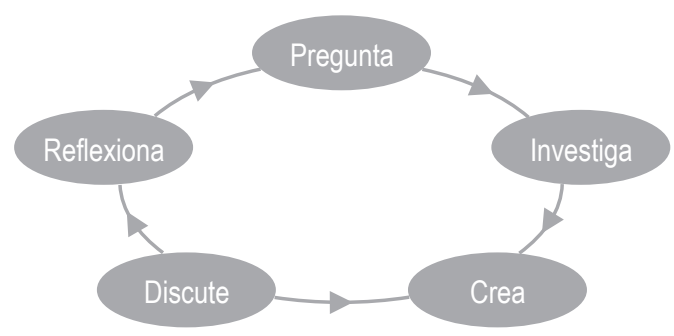

Figura 1: Ciclo de actividades IBL

Como puede apreciarse en la figura II, se diseñaron seis temas tratando de dar respuesta a grandes aspectos relacionados con la integración curricular de las TIC's en los centros educativos españoles. La principal ventaja que aporta esta forma de trabajo, constituida por una suerte de espiral circular, tiene que ver con la transferencia que el alumnado puede hacer de los contenidos procedimentales trabajados. Se mantiene una fórmula de trabajo consistente a lo largo de los 6 temas propuestos, a pesar de que sus contenidos y objetivos difieren sustancialmente.

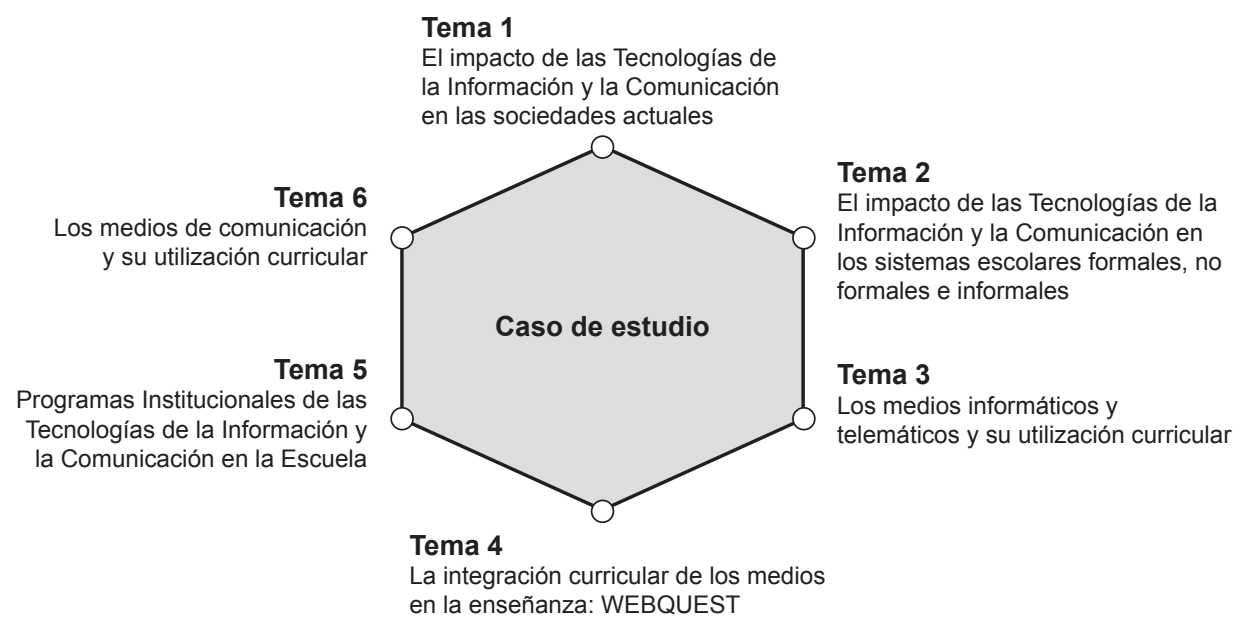

Figura 2: Esquema de contenidos de la asignatura

De esta forma, la asignatura quedó dividida en seis bloques de aproximadamente dos semanas de duración cada uno, a excepción del cuarto al que, por su especial complejidad se le dedicó el doble de tiempo.

Un aspecto relevante a destacar, estrechamente relacionado con las pretensiones de la asignatura de fomentar aprendizajes contextualizados, se encuentra en el hecho 
de que el alumnado siempre tiene como punto de referencia un caso de estudio para el que debe ir generando distintos artefactos educativos. El caso describe pormenorizadamente un centro educativo del que el alumnado de la asignatura forma parte. Se diseñó integrando un número significativo de características relevantes para el diseño educativo propuesto, tomando siempre como referencia centros escolares de la ciudad de Valladolid, bien conocidos por el profesorado.

\section{LO QUE EL OJO NO VE: TECNOLOGÍA DE APOYO AL PROCESO EDUCATIVO}

I: Bueno, ya estamos otra vez como cuando comenzamos a buscar tecnologías de apoyo para la asignatura. (Comenta Iván en tono jocoso)

B: Sí, parece claro que el diseño educativo que estamos proponiendo necesita otras cosas (tecnologías) que las que estamos usando ahora. ¿Se os ocurre qué podemos utilizar para darle soporte?

$R$ : No sé, hay muchas alternativas, pero creo que debemos decantarnos por el software libre. iAl final siempre tenemos que andar dependiendo de licencias $e$ historias que no hacen más que entorpecer!

I: Hummm. Sí, estoy de acuerdo contigo. iEs un rollo!... Por cierto, he visto cómo funciona Plone y creo que podría servirnos. He mirado el manual por encima, y además de ser libre, permite adaptar bastantes cosas.

B: iOye!, ¿y la herramienta que usa Chip? ¿cómo se llama...?

I: Sí, I-labs... La verdad es que nos vendría de maravilla una wiki de esas características, pero todavía no está disponible. Además no tiene un repositorio de información... no sé, de todas formas podemos preguntarle.

R: ¿Y moodle, o.LRN? ¿Parece que están pegando fuerte no?

B: Sí, eso parece, pero hasta que la universidad no adopte un campus virtual unificado, creo que es bastante arriesgado optar por tecnologías que obliguen a generar diseños educativos enlatados.

I: Ya... Pues no sé. Creo que debemos buscar opciones y compararlas... nos damos unos días y vemos...

Uno de los puntos críticos a los que Rocío y sus compañeros de asignatura tuvieron que hacer frente fue el de la selección de la tecnología de apoyo al proceso educativo. Hasta ese momento venían utilizando la plataforma Synergeia (Appelt \& Birlinghoven, 2001) (Itcole, 2005), pero a pesar de sus grandes potencialidades como entorno favorecedor de la colaboración, no resultaba suficiente. Su elevada flexibilidad hacía que el alumnado entendiese cada tema como disjunto del anterior, haciendo que el proceso educativo no fuese contemplado como un todo. Por este motivo analizaron y discutieron distintas posibilidades tecnológicas (Plone, i- 
labs, moodle, .LRN) decidiendo al final la integración de la tecnología wiki con la plataforma Synergeia que ya venían usando en cursos anteriores.

El primer WikiWikiWeb fue creado por Ward Cunningham en 1995, para generar un repositorio de patrones Portland (Portland Pattern Repository). En palabras del propio Cunningham, un wiki es "the simplest online database that could possibly work". No fue hasta enero del 2001 cuando Jimbo Wales y Larry Sanger decidieron utilizar un wiki como base para el proyecto de la conocida enciclopedia Wikipedia. Para esta ambiciosa tarea crearon un software propio denominado MediaWiki, adoptado después por muchos otros wikis. Puesto que Wikipedia es un recurso ampliamente conocido por el alumnado de NNTT, además de ser muy fácilmente usable, se eligió como tecnología básica de diseño del entorno wiki de soporte a la asignatura. La principal ventaja que tiene un wiki con respecto a otras tecnologías web, es que permite crear y mejorar las páginas de forma instantánea, dando una gran libertad al usuario, todo ello a través de una interfaz extremadamente sencilla. Una de las principales ventajas que aporta el uso de esta tecnología para desarrollar los temas de la asignatura siguiendo el modelo del IBL, es que permite la escritura de documentos colectivamente por medio de un lenguaje de wikitexto editado mediante un navegador.

No obstante, esta tecnología no aportaba todos los requisitos que el diseño educativo de la asignatura requería. El profesorado consideró necesario disponer de un entorno privado y accesible únicamente al alumnado matriculado para generar y compartir la información "sensible" que se generase durante el curso. Por este motivo se optó por seguir utilizando Synergeia como repositorio de información y lugar para establecer debates asíncronos privados alrededor de la fase "discute" de cada tema. De esta manera, algunos espacios de la wiki apuntaban a entornos cerrados gestionados dentro de Synergeia.

Además de estas dos herramientas y como consecuencia de la relevancia que las dinámicas de tutoría tienen en los escenarios CSCL (Jorrín, 2006) se pensó en la utilización de dos herramientas diseñadas en el seno del grupo GSIC-EMIC: Quest y Samsa.

Quest (Gómez et al, 2002) es un software desarrollado en el mencionado grupo de investigación que permite automatizar el ciclo de vida completo de cuestionarios. Entre sus funcionalidades se encuentran la edición de cuestionarios, su publicación en web, y la contestación de los mismos también vía web. A su vez, permite almacenar las respuestas que los usuarios hayan aportado, procesando de diversas maneras las 
respuestas. Por ejemplo, genera tablas que recogen las respuestas vertidas por el alumnado en diversos momentos del proceso educativo. Estas tablas son procesadas por el profesorado para después promover sesiones de debate, tratando de incidir en los aspectos más relevantes abordados durante las clases previas. Esta herramienta permite realizar, a su vez, un seguimiento pormenorizado de los avances conseguidos por los grupos de trabajo dentro de la asignatura, favoreciendo notablemente la tutoría y evaluación continua.

Con el objetivo de dinamizar aún más estos procesos de tutoría, se empleó también SAMSA (System for Adjacency Matrix and Sociogram-based Analysis) (MartínezMonés et al, 2003), un software que apoya el análisis de redes sociales. Contiene varios módulos de entrada, que toman datos de diferentes fuentes, en nuestro caso de los logs de eventos que almacenan automáticamente tanto el espacio wiki como Synergeia. Samsa construye una matriz que representa la red de interacciones producida en estos dos espacios, y calcula las medidas elegidas por el profesorado, para posteriormente generar un fichero en formato DL (Data Language). Este formato es aceptado por UCINET (Borgatti et al, 1999) un paquete software genérico para análisis de redes sociales. Un ejemplo del resultado generado puede verse en la figura 3. Este tipo de redes sociales le sirvieron a Rocío para preparar y dinamizar las tutorías específicas que mantuvo con todos los grupos de trabajo en dos momentos del curso.

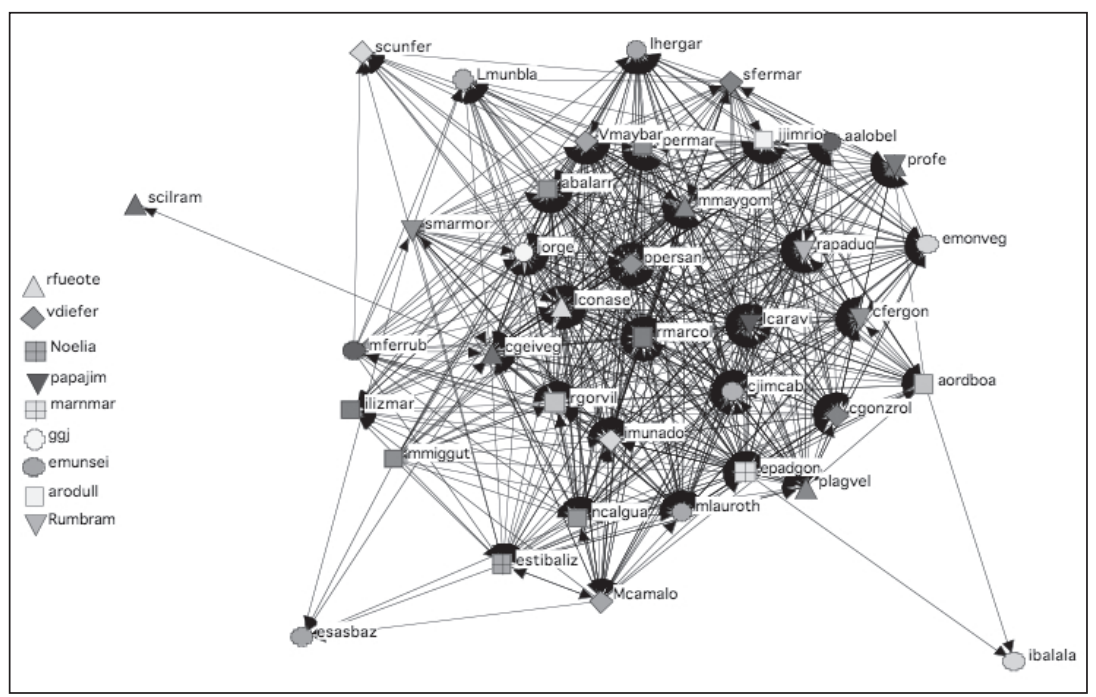

Figura 3: Sociograma de participación del alumnado durante los dos primeros meses de clase 
La confluencia de estas cuatro tecnologías permitió a Rocío generar un espacio de trabajo único aglutinando el diseño completo de aprendizaje, y facilitando de esta forma lo que denominamos como co(wiki)laboración. El resultado del entorno al que el alumnado tuvo acceso (figura 4) está accesible en http://hera.fed.uva.es/wiki. Se puede observar cómo se integraron cuatro tecnologías distintas de forma transparente al alumnado, permitiendo de esta forma que la tecnología de soporte, "lo que el ojo no ve", se convirtiera en una respuesta adaptada a las peculiaridades y necesidades de un proceso educativo basado en la colaboración mediada por tecnología.

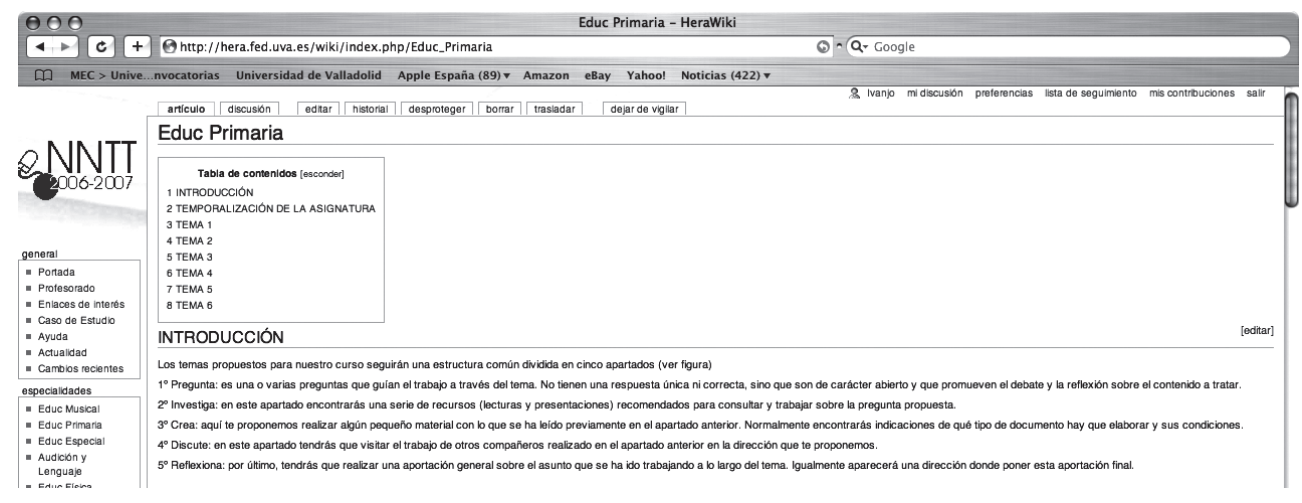

Figura 4: Aspecto del entorno colaborativo diseñado para la experiencia

\section{LO QUE EL OJO NO VE: PROCESO DE EVALUACIÓN}

De forma paralela al devenir educativo descrito, Rocío y sus compañeros pusieron en práctica un proceso de evaluación de la asignatura que les permitiera valorar la calidad de la experiencia que estaban desarrollando.

R: Como ya viene siendo habitual, ahora le toca el turno a la evaluación. Creo que deberíamos generar un documento con la planificación y la distribución de tiempos y tareas.

B: Sí, así cada cual sabremos en qué momento de la asignatura estamos y qué tareas de evaluación toca hacer. ¿Vamos a continuar usando el método mixto?

R: Yo creo que sí. A pesar de que es muy costoso, funciona bastante bien y siempre nos aporta soluciones de mejora.

I: Sí, yo también lo creo. De todas formas, debemos ajustarlo al tiempo que tenemos y a las personas de apoyo. ¿No creéis?

B: Tal vez con tres cuestionarios y una observación por semana a lo largo del curso sea bastante... 
R: Sí, pero lo que también tenemos que hacer es delimitar el tiempo que tardaremos en analizar la información recogida, porque hay veces que no da tiempo a preparar la siguiente tarea...

Durante las semanas previas al inicio de la asignatura se sucedieron múltiples reuniones en torno a la evaluación que se deseaba implementar. Una de las cuestiones que todo el mundo tuvo clara fue la relativa a la aplicación del método mixto de evaluación (Martínez et al, 2003, 2005) propuesto años atrás dentro del grupo GSIC-EMIC. La elección de este modelo de evaluación vino determinada, sin duda, porque ya se había puesto en práctica en experiencias anteriores, dando grandes resultados.

Como muestra la figura 5, el proceso de evaluación se encuentra repartido en torno a las tres fases en las que se divide el método mixto, a saber; Fase de preparación; Fase durante el desarrollo del curso y; Fase Final. A lo largo de las tres fases se preparan, y se ponen en práctica las cuatro técnicas de recogida de información utilizadas; Recogida automática de eventos (cada día se genera automáticamente un informe); observaciones (diez observaciones a lo largo del curso); cuestionarios (tres cuestionarios; inicial, intermedio y final); y grupos de debate (Inicial, intermedio y final). Éstas técnicas se dividen en cuantitativas y cualitativas (de ahí la denominación del método empleado). Dentro del primer grupo se encuentra únicamente la recogida automática de logs de eventos provenientes de las interacciones que el alumnado genera dentro del espacio Wiki y de la plataforma colaborativa Synergeia, usadas en la asignatura. Sin embargo, dentro del segundo grupo de técnicas se encuentran los cuestionarios, las observaciones y los grupos de debate. De esta manera el método va generando dos líneas de datos, una línea con los cuantitativos y otra más densa con los cualitativos.

El proceso de análisis de los datos resulta especialmente complejo, ya que en función de su origen y tipología, se someten a tres etapas de análisis en paralelo.

Los datos automáticos recogidos, se analizan con la herramienta SAMSA. Obteniéndose así, un análisis de las redes sociales generadas en el curso.

En segundo lugar, el conjunto de datos cualitativos obtenidos en las observaciones, en los cuestionarios y en los grupos de debate, se procesan mediante la herramienta de análisis cualitativo Nud*ist Vivo (QSR, 1997), obteniendo sendos informes articulados en torno al esquema de categorías de análisis previamente definido. 
En tercer lugar, los datos cuantitativos provenientes de los cuestionarios realizados, se someten a un análisis estadístico descriptivo, del que se obtienen datos procesados que apoyan el resto de datos del proceso.

Todos los datos analizados de estas tres formas, se integran en un informe parcial de conclusiones, que aporta retroalimentación al proceso de cara al curso siguiente en el que se vuelve a realizar una segunda iteración de todo el método. La suma de los cuatro informes parciales realizados, conforman el grueso de los resultados que se han obtenido.

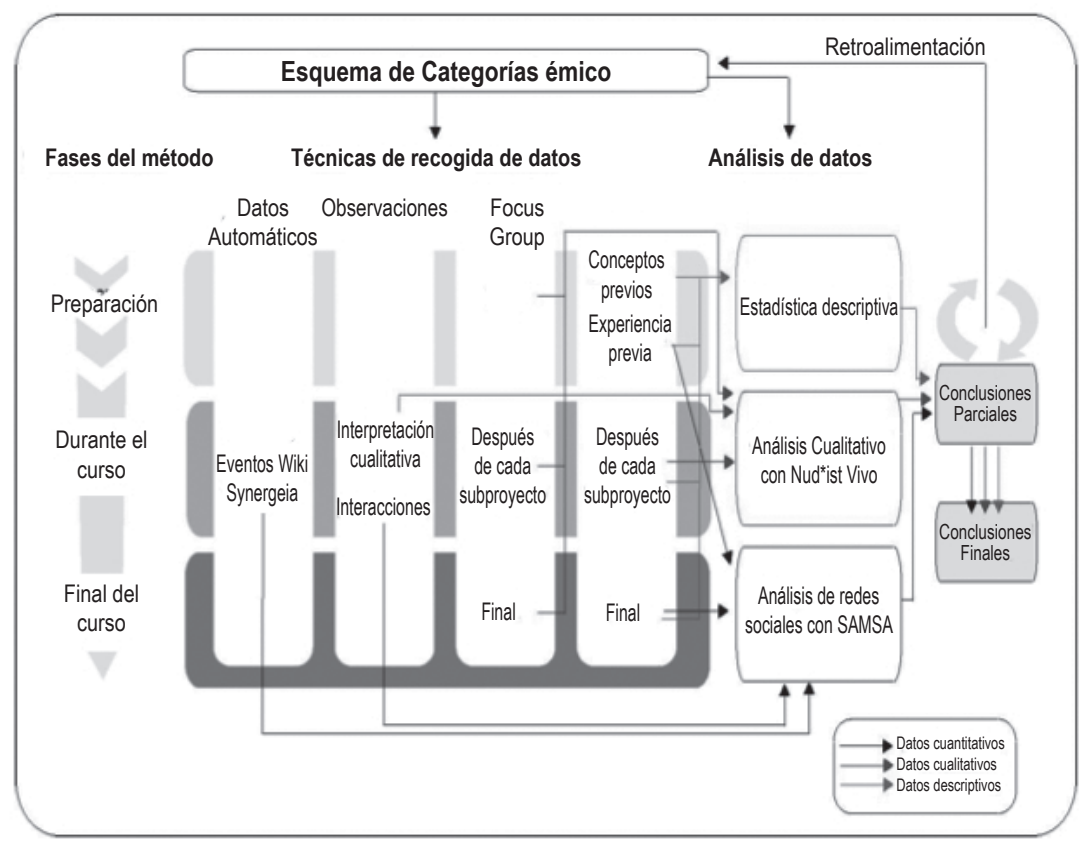

Figura 5: Esquema de evaluación del caso

El método provee una triangulación severa de datos y fuentes, aportando credibilidad a los resultados obtenidos. Tal vez esta cuestión resulte uno de los principales puntos fuertes de la experiencia, aportándole un gran valor añadido. No obstante, es necesario apuntar que el método genera una carga de trabajo tan extensa, y resulta tan laborioso que se hace inabarcable para un único investigador. En la asignatura de NNTT se ha podido llevar a buen fin por la profusa colaboración de un núcleo de cinco personas del grupo de investigación GSIC-EMIC, así como por la desinteresada participación del alumnado de la asignatura de NNTT. 


\section{CONCLUSIONES Y TRABAJO FUTURO}

Son numerosas las conclusiones que se han generado tras el desarrollo de la experiencia en la asignatura de NNTT. A pesar de que el curso puesto en marcha no ha sido demasiado extenso (un cuatrimestre) la exhaustividad de la evaluación realizada han ayudado a Rocío y sus colaboradores a extraer algunos puntos fuertes y débiles, que detallamos a continuación.

- Las tecnologías de apoyo al proceso educativo son valoradas muy positivamente tanto por Rocío como por su alumnado.

B: ¿Qué os parecen las tecnologías que estamos utilizando en la asignatura? Synergeia, Quest, la Wiki, ¿Cómo las véis?

C: bien. A mí lo que me gusta es que puedes, o sea, que lo puedes subir ahí todo y lo puedes mirar desde cualquier ordenador. Vamos, que no tienes que venir al ordenador de aquí, del aula para mirarlo, porque así yo que sé, te puedes ir a tu casa, y quedar con el compañero y arreglarlo o cualquier cosa

R: Bueno, la wiki es un poco más fácil de usar que synergeia, pero bueno, hasta que le coges un poco el aire a la metodología de funcionamiento. Pero en cuanto lo coges, (comenta entre risas) no tienes que hacerte ni un esquema, ni un croquis...

M: Creo que son bastante didácticos. Por ejemplo la wiki, pues te va explicando cuáles son los puntos en los que te debes fijar, y dónde tienes que poner las cosas.

El diálogo anterior, mantenido con cuatro alumnos voluntarios del curso durante un grupo de debate el día 28/09/2006 puede servir de muestra para conocer la valoración que el alumnado de la asignatura hace de las tecnologías empleadas para dar soporte al proceso educativo. El alumnado realza la capacidad de guiado y organización que el espacio tecnológico diseñado proveía. En esta misma línea se encuentra la siguiente valoración realizada por Rocío en su cuaderno de campo al finalizar el curso.

"Si en el papel la wikiweb ya me pareció una gran idea cuando la estuvimos diseñando, por lo que tenía de globalizado, tanto en la visión de toda la asignatura como en el procedimiento didáctico, a la hora de ponerla en marcha, como profe ha sido una auténtica gozada (nos olvidamos de los problemas con el servidor las dos primeras semanas, que eran otros que no vienen al caso). La wiki permite cambiar todo sobre la marcha, con lo cual, cualquier tipo de acuerdo al que se llega en clase, cualquier enlace que no está bien puesto o no funciona como debería, el ir añadiendo comentarios a la parte del reflexiona prácticamente en tiempo real en clase, el poner las notas en el mismo espacio de trabajo,... La verdad es que 
todo han sido facilidades para trabajar y la combinación con synergeia, ahora sí utilizada sobre todo como repositorio de documentos, tanto para el profe como para el alumnado, ha sido de una facilidad enorme. He de decir que he podido ser totalmente autónoma en el plano tecnológico todo el curso, lo cual no era fácil usando una nueva herramienta telemática".

- El diseño cíclico de cada tema, apoyado en los principios del aprendizaje por indagación, además de ayudar al alumnado a aprender, ha generado un proceso de evaluación justo y equilibrado. Mostramos a continuación la reflexión generada por Rocío en su cuaderno de campo al respecto.

“...Una cosa que me ha sorprendido al final del curso es que las notas que han podido sacar los alumnos este año han sido sensiblemente más altas que las de otros años. Mi expectativa era justo la contraria, por aquello de que cuando empiezas a fragmentar una nota en cosas pequeñas se van perdiendo décimas que luego hacen que nunca se llegue al sobresaliente.

Este sistema de evaluación continua por temas es más ajustado de lo que yo preveía al comienzo, y realmente permite al alumnado ir sacando en cada tema entre un 70 y un $90 \%$ de la nota asignada, con lo cual, al final todas las notas han estado entre el 7 y el 9'5. !Hacía años que no daba tantos sobresalientes en un grupo, la friolera de 9!"

- $\quad$ El diseño de la asignatura ha fomentado la colaboración entre el alumnado, y éste la valora muy positivamente. A continuación mostramos un extracto del segundo grupo de debate realizado, como ejemplo de esta cuestión.

C: A mi me gusta la dinámica que estamos siguiendo en clase... que sea en parejas, hacer el trabajo, y después comentarlo con tus compañeros y que ellos opinen, y tú opinas. A mi eso me gusta.

B: ¿Por qué te gusta colaborar?

C: Si.A mi sí

B: ¿Y te gusta que...?

C: Y me gusta que colabore todo el mundo. No que solamente colabore yo. Si no que seamos una pareja y que yo haga una parte del trabajo y que ella haga otra, no que me toque a mí entero, o a ella entero. No, eso no. O sea si somos un grupo, pues hay que trabajar como un grupo, porque yo creo que todos hemos vivido experiencias en las que nos ha tocado hacer entero el trabajo o que no hemos hecho nada del trabajo, y eso no es así, o sea, compañerismo ino? 
- Las tutorías se han convertido en una parte esencial del diseño. La herramienta para generar redes sociales ha ayudado sobremanera a este propósito. A continuación aportamos un ejemplo de los grafos con los que contaba Rocío para guiar y mediar en el aprendizaje de su alumnado durante las tutorías desarrolladas en el curso.

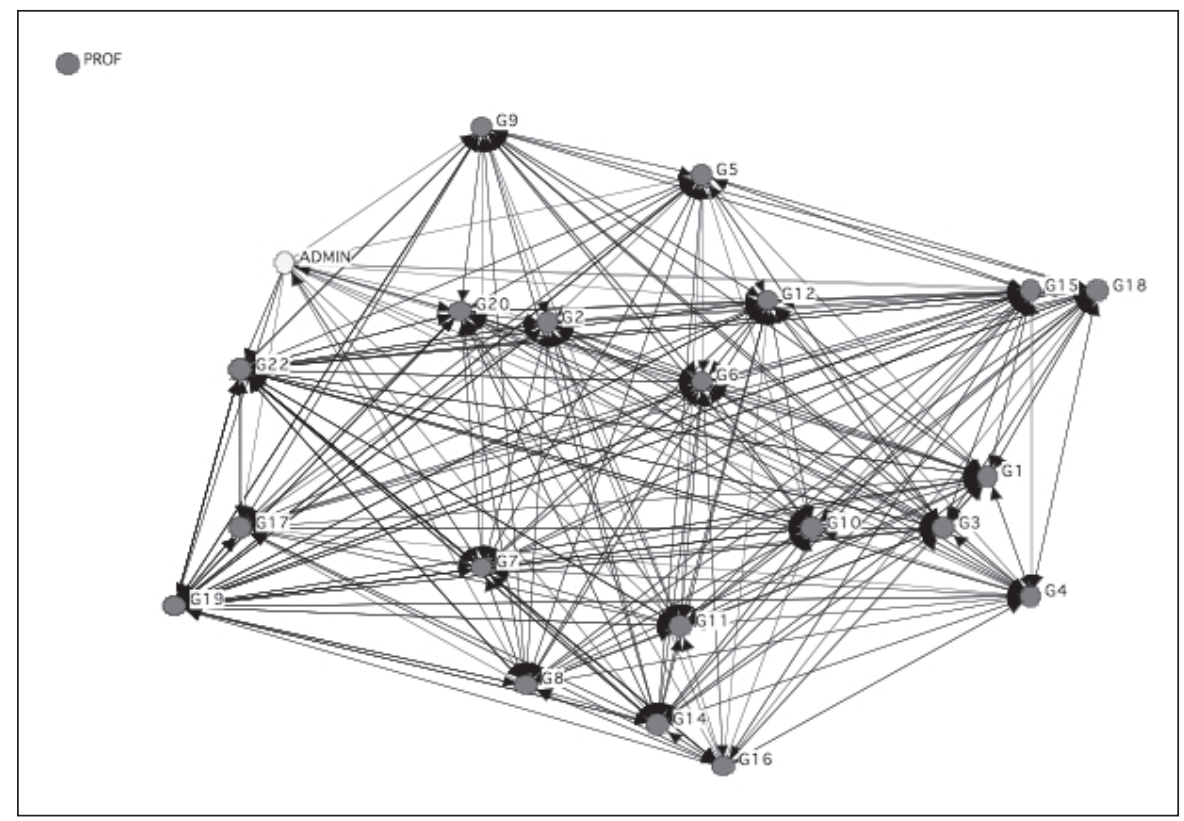

Figura 6: Sociograma asociado a la primera fase, con los alumnos/as agrupados por parejas, con el nombre del grupo asignado, e incluyendo a la profesora

En la figura 6 se muestra la red social formada por la interacción de las distintas parejas de trabajo durante los primeros temas del curso. Esta información fue utilizada por Rocío para mostrar al alumnado el lugar en el que se encontraba dentro de la red social, y así discutir, y mejorar el trabajo de cada pareja en subsiguientes temas, nunca para establecer procesos sancionadores 


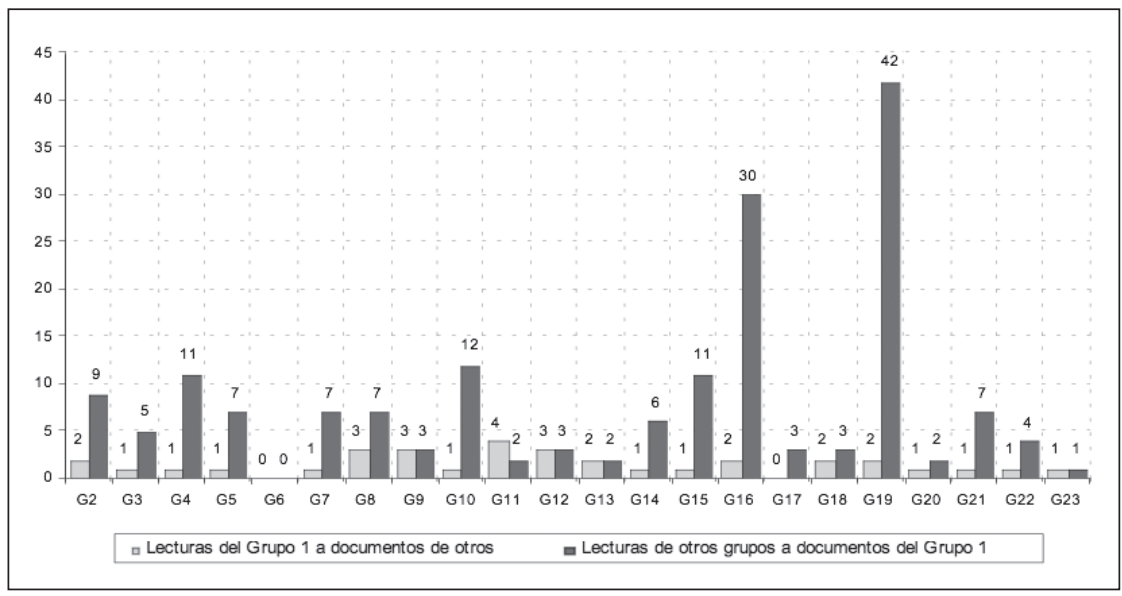

Figura 7: Diagrama de representación del número de documentos consultados por el grupo 1 y de los documentos generados por el mismo grupo que fueron consultados por otros grupos

La figura 7 muestra un histograma que recoge la comparación entre las lecturas realizadas por los componentes de la pareja 1 a documentos generados por sus compañeros/as de clase, y los documentos creados por ellos mismos que fueron leídos por otras parejas del curso. Esta información fue utilizada por Rocío en las tutorías para conocer el por qué de la lectura de unos u otros documentos, contrastando las lecturas a documentos con la calidad de los mismos.

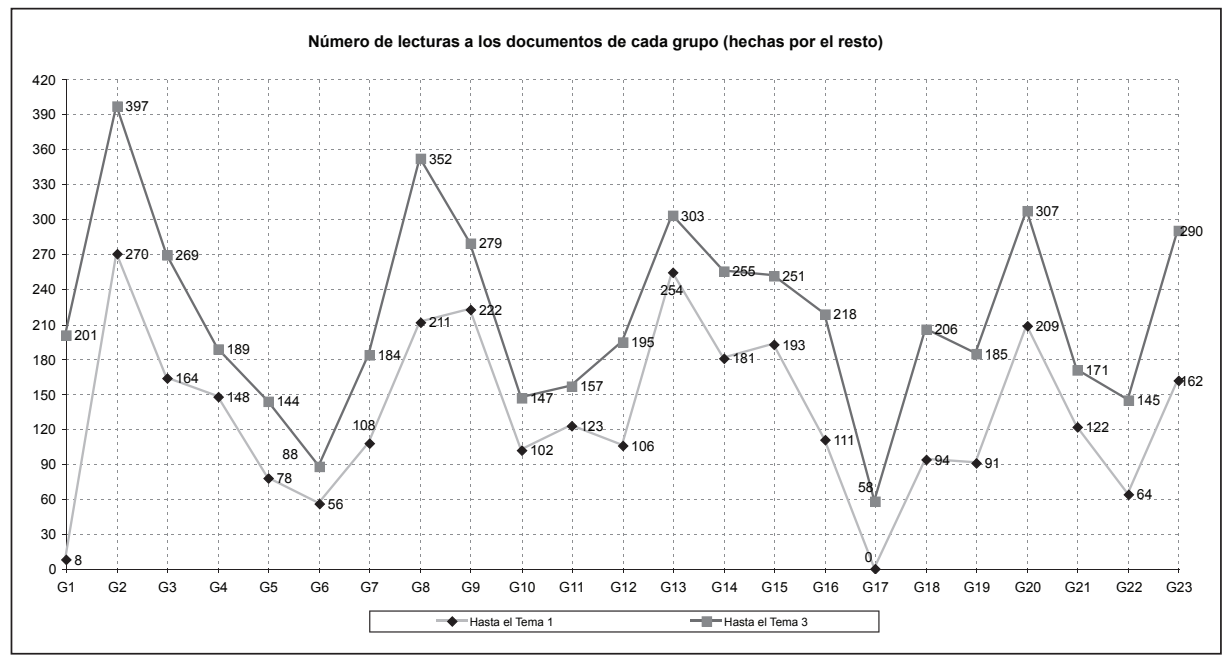

Figura 8: Comparación de lectura de documentos por parte del grupo 1 hasta los temas 1 y 3 
Por su parte, la figura 8 muestra una comparación de la evolución de lecturas de documentos realizadas por el grupo de trabajo 1 hasta la finalización de los temas 1 y 3. Esta información fue usada por Rocío en las tutorías para discutir con cada uno de los grupos la evolución de su trabajo a lo largo del curso.

Después de comentar brevemente algunos ejemplos de redes sociales y gráficos generados automáticamente a partir de las informaciones extraídas del espacio Wiki y de Synergeia, podemos comprobar cómo los datos obtenidos facilitaron la labor de la profesora del curso en los procesos de tutoría individualizada.

El proceso de evaluación de la asignatura también ha dejado al descubierto algunas carencias y puntos débiles que deberán ser subsanados. Entre ellos se encuentran los siguientes:

- Como sucede en todo proceso educativo apoyado por tecnología, las clases están sujetas al correcto funcionamiento del aula destinada al efecto. Durante la experiencia, Rocío ha extraído algunos principios de procedimiento esenciales para que el diseño educativo prospere adecuadamente.

"Una lección a aprender es que no se puede llegar al aula on time, lo suyo es irse un buen rato antes, abrir todos los ordenadores (incluido el del profe, que funciona como le viene en gana) y ponerlos a funcionar para que cuando la gente llegue a clase no se pierdan los 10 primeros minutos en problemas técnicos de toda índole. Empezar la clase explicando qué se va a hacer ese día sin que todo el mundo esté ya en red es una fuente de interrupciones y de idas y venidas que no se centran en el contenido de lo que estás explicando, con lo cual muchas veces hay que volver a dar las explicaciones varias veces".

- Otra lección aprendida durante el curso ha sido que debemos poner mayor énfasis en los aspectos puramente educativos de la asignatura, puesto que no deseamos que el alumnado se quede únicamente con las cuestiones prácticas de uso de la tecnología.

"El último día de clase les comento de manera informal y según van saliendo del aula que ya es la última vez que nos vemos y dónde estoy en la facultad. Algunos se quedan asombrados de que sea el departamento de Pedagogía y no el de informática. Yo les aclaro que esta asignatura pertenece a esa área de conocimiento y que somos pedagogos los que la llevamos. Este asombro me pone en la pista de que, en realidad, una parte importante del alumnado sólo se queda con la idea del 
manejo de las máquinas o de determinados programas informáticos y no con la idea educativa que queremos transmitir".

- A pesar de que el alumnado elige como recurso tecnológico más utilizado durante la asignatura Synergeia (90,90\% frente al 81,81\% del espacio Wiki), resulta paradójico comprobar que cuando se les pregunta por la facilidad de uso, consideran que el espacio Wiki es el más sencillo de utilizar (el espacio Wiki es valorado con un 5.32 de media y una D.T. de 2.13, mientras que Synergeia es valorada por el grupo con una media de facilidad de uso de 5.5 con una D.T. de 2.33). Este hecho nos hace pensar que tal vez sea necesario modificar bien las propiedades de uso de la plataforma Synergeia, bien la plataforma completa, eligiendo otra que facilite más el trabajo en la asignatura.

Para concluir con este apartado, y dando respuesta a los principales problemas detectados en la asignatura de NNTT antes de la puesta en práctica de ésta experiencia, podemos decir que en general se ha reducido la carga de trabajo que acarreaba la asignatura tanto para el alumnado como para el profesorado. A su vez, hemos comprobado que la distribución del alumnado en grupos de trabajo homogéneos (parejas y grupos de cuatro) que no cambiaban durante el curso ha mejorado sustancialmente la dinámica de clase simplificándola. También hemos observado que el espacio Wiki se ha convertido eminentemente en un guión de aprendizaje que ha contribuido a un mejor desarrollo del programa de la asignatura. Otro aspecto relevante a destacar tiene que ver con que se ha reducido la parte teórica de la asignatura, aumentando el tiempo que el alumnado podía dedicar a la aplicación práctica de los contenidos teóricos trabajados. Por último podemos afirmar que se ha conseguido que el alumnado entendiese como una unidad los recursos tecnológicos de apoyo empleados, dinamizando de esta forma el desarrollo de la asignatura.

Puesto que la experiencia ha resultado altamente satisfactoria, en la actualidad continuamos poniendo en práctica el diseño propuesto para la asignatura en otros cursos de similares características. También nos encontramos inmersos en una transversalización de la innovación propuesta en la asignatura de NNTT. Pretendemos extender la experiencia, usando como base de apoyo la tecnología wiki, a un conjunto de asignaturas integradas dentro de la misma titulación. Ello permitirá al alumnado utilizar una misma plataforma de colaboración para un número significativo de las asignaturas que conforman su titulación. Para poder dar respuesta a esta propuesta se requiere de aulas dotadas con equipos informáticos. Como en las facultades españolas no suele ser habitual encontrar equipos dentro de las aulas ordinarias, hemos optado por la compra de una aula móvil dotada con 16 equipos portátiles 
que permitirán al profesorado y al alumnado convertir cualquier aula en un espacio colaborativo mediado por tecnología.

"El último día de clase, en ese momento informal de las despedidas, algunos alumnos me dicen que han aprendido a hacer muchas cosas y que les ha gustado la asignatura, lo cual es la mejor evaluación que te pueden hacer. Creo que el ambiente general al final es bastante distendido y bueno entre el alumnado y de ellos conmigo".

Rocío

\section{NOTAS}

1 Information and Communication Technologies

2 Grupo de Sistemas Inteligentes y Cooperativos-Educación Medios Informática y Cultura (http://ulises.tel.uva.es/)

3 Professor, Library \& Information Science. U. of Illinois at Urbana-Champaign. Autor de "The Inquiry page" (http://inquiry.uiuc.edu/)

\section{REFERENCIAS BIBLIOGRÁFICAS}

Appelt, W.; Birlinghoven, S. (2001). What groupwaredousersreallyuse?Analysis of the usage of the BSCW system. [en línea] Disponible en: http://bscw.gmd. de/Papers/PDP2001/PDP2001.pdf [consulta 2006, 7 de mayo]

Bologna Declaration (1999) [en línea] Disponible en: http://www.mec.es/ univ/html/informes/EEES 2003/ Declaracion Bolonia.pdf [consulta 2006, 7 de mayo]

Borgatti, S.; Everett, M.; Freeman, L. (Eds.) (1999). UCINET 5.O Version 1.00. Natick: Analytic Technologies.

Bruce, B. C. (2000). Credibility of the web: Why we need dialectical reading. Journal of Philosophy of Education (special issue), 34(1), 97-109.

ITCOLE Research Project. Synergeia Website. Retrieved in December, 2005 from. [en línea] Disponible en: http:// bscl.gmd.de [consulta 2006, 7 de octubre]

Rubia Avi B.; Anguita Martínez R.; Jorrín Abellán, I. M.; Ruíz Jorrín-Abellán, I. M. (2006) Formative portrayals emerging in CSCL environments: A case study (in spanish). Phd dissertation, Faculty of Education, Department of Pedagogy, University of Valladolid, Spain, May 2006.

Koschman, T. (1996). CSCL: Theory and Practice of an Emerging Paradigm Mahwah, N. J. Lawrence Erlbaum.

Martínez, A.; Dimitriadis, Y.; De la Fuente, P. (2003). Interaction analysis for formative evaluation in CSCL. Llamas, M.; Fernández, M. J.; Anido, L. E. (Ed.). Computers and Education. Towar a lifelong learning society. Kluwer Academic; 227-238. 
Martínez, A.; Gómez, E.; Dimitriadis, Y.; Rubia, B.; Jorrín, I.; Vega, G. (2005): Multiple case studies to enhance project-based learning in a Computer Architecture course. IEEE Transactions on Education. Vol: 48, 482- 490

QSR. (1997). NUD*IST. Software for qualitative data analysis. Thousand Oaks, CA, USA: Scolari.
Rubia, B.; Anguita, R.; Jorrín, I. M.; Requies, I. (2006). La formación práctica interdisciplinar de magisterio en un entorno tecnológico y colaborativo: dos años de experiencia. En Rodríguez Sumaza, C.; de La Calle Velasco, M. J. (Coords.). La Innovación Docente ante el Espacio Europeo de Educación Superior. Universidad de Valladolid. 371-382.

\section{PERFIL ACADÉMICO Y PROFESIONAL DE LOS AUTORES}

Iván M. Jorrín Abellán es Profesor del Departamento de Pedagogía de la Universidad de Valladolid, y doctor por esta misma universidad. En la actualidad centra sus esfuerzos de investigación en las implicaciones educativas de los espacios de enseñanza-aprendizaje basados en los principios del CSCL. Sus trabajos más recientes hacen hincapié en el desarrollo de un marco de evaluación cualitativa aplicable a estos entornos. Ha participado en diversos proyectos y publicaciones en el seno del grupo GSIC-EMIC pudiéndose consultar los más destacados en http:// gsic.tel.uva.es/members/ivanjo.

Rocío Anguita Martínez es Profesora del Departamento de Pedagogía de la Universidad de Valladolid, doctora por la Universidad de Granada y miembro del grupo GSIC-EMIC. Pertenece al KALEIDOSCOPE, Red Europea para la innovación científica en materia de Tecnologías para la Educación y dentro del grupo centrado en el CSCL (Computer Supported Collaborative Learning). Su segunda línea de investigación se centra en el campo de las TIC's y el género. Ha participado en multitud de publicaciones y eventos, pudiéndose consultar los más destacados en http://ulises.tel.uva.es/members/rangmar.

Bartolomé Rubia Avi es Profesor del Departamento de Pedagogía de la Universidad de Valladolid, siendo doctor por esta Universidad en Diseño Curricular e Investigación Educativa. Miembro del Grupo GSIC-EMIC laboratorio asociado al KALEIDOSCOPE, Red Europea para la innovación científica en materia de Tecnologías para la Educación y dentro del grupo centrado en el CSCL (Computer Supported Collaborative Learning). Ha participado en multitud de publicaciones y eventos, pudiéndose consultar los más destacados en http://ulises.tel.uva.es/ members/brubia. 
Yannis Dimitriadis es profesor titular en la Escuela Técnica Superior de Ingenieros de Telecomunicación de la Universidad de Valladolid. Ingeniero por la Universidad Nacional de Atenas (1981), M.S. por la Universidad de Virginia (1983) y doctor por la Universidad de Valladolid en dos ocasiones (1992 y 1995). Es investigador principal del grupo GSIC-EMIC y sus intereses de investigación se centran en el Computer supported Collaborative Learning y en los Sistemas distribuidos. Ha participado en multitud de publicaciones y eventos, pudiéndose consultar los más destacados en http://ulises.tel.uva.es/personal/yannis.

Inés Ruíz Requies es profesora asociada y personal investigador del Departamento de Pedagogía de la Universidad de Valladolid y miembro del Grupo GSIC-EMIC. En la actualidad se encuentra realizando la tesis doctoral sobre "La formación docente en entornos CSCL: de la habilidades sociales a la propuesta curricular". Ha participado en proyectos y publicaciones relacionadas con la Tecnología Educativa, pudiéndose consultar en http://ulises.tel.uva.es/personal/ irure.

Jose Antonio Marcos es profesor ayudante en la Escuela Técnica Superior de Ingenieros de Telecomunicación de la Universidad de Valladolid. Es miembro del grupo GSIC-EMIC. Pertenece al KALEIDOSCOPE, Red Europea para la innovación científica en materia de Tecnologías para la Educación, dentro del grupo centrado en el CSCL (Computer Supported Collaborative Learning). En la actualidad se encuentra realizando su tesis doctoral sobre "Análisis de interacciones en los entornos CSCL".

Sara Villagrá Sobrino es personal investigador del Departamento de Pedagogía de la Universidad de Valladolid y miembro del Grupo GSIC-EMIC. En la actualidad se encuentra realizando los cursos de doctorado en la Facultad de Educación y Trabajo Social en el Departamento de Pedagogía.

Fecha de recepción del artículo: 11/06/07

Fecha de aceptación del artículo: 17/09/07 